\title{
Free-volume studies in polyurethane membranes by positron annihilation spectroscopy
}

\author{
M.F. Ferreira Marques ${ }^{\mathrm{a}, \mathrm{b}, *}$, C. Lopes Gil ${ }^{\mathrm{b}}$, P.M. Gordo ${ }^{\mathrm{b}}$, Zs. Kajcsos ${ }^{\mathrm{b}, \mathrm{c}}$, \\ A.P. de Lima ${ }^{b}$, D.P. Queiroz ${ }^{\mathrm{d}}$, M.N. de Pinho ${ }^{\mathrm{d}}$ \\ ${ }^{a}$ Instituto Superior de Engenharia, 3031-199 Coimbra, Portugal \\ ${ }^{\mathrm{b}}$ ICEMS, Departamento de Física, Universidade de Coimbra, 3004-516 Coimbra, Portugal \\ ${ }^{\mathrm{c}}$ KFKI, Research Institute for Particle and Nuclear Physics, H-1525 Budapest P.O.B. 49, Hungary \\ ${ }^{\mathrm{d}}$ Departamento de Engenharia Química, Instituto Superior Técnico, Av. Rovisco Pais 1, 1049-001 Lisboa, Portugal
}

\begin{abstract}
The free-volume parameters in various urethane/urea membranes obtained by varying the ratio of the structural constituents, polypropylene oxide and polybutadiene, were studied by positron lifetime and Doppler broadening measurements. On bi-soft segment membranes, a correlation was found between the composition of membranes, the normalized free volume, the radii of the holes and gas permeability. However, the correlation is not clear when PU data are also considered, indicating that other features must also play an important role in the permeation mechanism.
\end{abstract}

(C) 2003 Elsevier Science Ltd. All rights reserved.

Keywords: Positronium; Urethane/urea polymers; Phase separation; Permeability; Pore size; Free volume

\section{Introduction}

Polyurethanes, extensively used in the chemical industry, usually consist of hard segments (urethane/ urea groups and/or low molecular weight chain extenders) and one or more soft segments (e.g., polyether/ polyesters). Depending on the processing parameters and on the type of hard and soft segments these can be totally mixed or phase-separated to different degrees (Cooper and Tobolsky, 1966; Blackwell and Gardner, 1979; Fridman and Thomas, 1980). This structure versatility enables the production of materials with a wide range of properties. One important application of urethane/urea membranes is the exploitation of their selective permeability in industrial separations, namely gas permeation and pervaporation.

\footnotetext{
*Corresponding author. Instituto Superior de Engenharia, 3031-199 Coimbra, Portugal. Tel.: +351-239-829-158; fax: +351-239-410-600.

E-mail address: fatima@lipc.fis.uc.pt (M.F. Ferreira Marques).
}

The free-volume concept is extensively adopted in polymer science to explain many properties at microscopic structural level and to relate them with macroscopic properties. Positron annihilation lifetime spectroscopy is an important method for studying subnanometer size holes and for the determination of their size distribution and free-volume fractions (e.g. Jean, 1993; Dlubek et al., 2002). In a polymer matrix, thermalized positrons can form positronium (Ps) that will be localized in free-volume holes. The primary annihilation mechanism of the long-lived triplet state of positronium (ortho-positronium, $o$-Ps) is the pick-off annihilation with electrons of the host medium, with annihilation parameters dependent on the physicochemical properties. The lifetime and intensity of this component can be translated into hole size and density of holes. Recently, it has been shown that the Doppler broadening method may also give an important contribution to these studies (e.g. Dlubek et al., 2000). Indeed, the $W$-parameter, extensively applied in solidstate physics investigations (Flores et al., 2002) seems to provide specific sensitivity to the molecular bounds and ordering of the polymer chains and for this reason the 
Doppler spectroscopy gains increasing importance and acceptance in polymer studies as well (e.g., Semaan et al., 2001).

In this work, urea/urethane membranes obtained through the introduction of two soft segments were studied with the aims of establishing how the freevolume hole properties are influenced by the phase separation or mixing of the polymer components and correlate them with the known permeability features.

\section{Experimental}

The polymer membranes were synthesized from two basic units: polypropylene oxide-based prepolymer with three isocyanate terminal groups (PU), supplied by Portuguese Hoechst, and polybutadiene diol (PBDO) from Aldrich, containing 20-30 wt \% vinyl, $10-25 \mathrm{wt} \%$ cis-1,4 and $50-60 \mathrm{wt} \%$ trans-1,4 isomers. Dibutyltin dilauryate (DBTDL), supplied by Aldrich, was used as catalyst. Toluene, from Merck, was used as solvent.

Cross-linked membranes were prepared through the reaction of the isocyanate groups of $\mathrm{PU}$ with the hydroxyl groups of PBDO catalyzed by DBTDL at $70-80^{\circ} \mathrm{C}$. The ratio of PU to PBDO was varied to yield cross-linked polymers with different compositions.

Measurements of positron lifetime (LT) were carried out on PU/PBDO samples with up to $75 \%$ PBDO. The LT spectra were recorded using a fast-fast time spectrometer (with $\mathrm{BaF}_{2}$ and Pilot-U scintillators), with a time resolution of $220 \mathrm{ps}$ FWHM for ${ }^{60} \mathrm{Co}$. The positron source, ca. $7 \times 10^{5} \mathrm{~Bq}$ of ${ }^{22} \mathrm{Na}$ was sandwiched between two identical specimens cut from $2 \mathrm{~mm}$ thick polymer sheets. The LT spectra, with about $1.7 \times 10^{6}$ integral counts, were recorded at $(298 \pm 0.5) \mathrm{K}$ and fitted by applying a version of POSITRONFIT (Kirkegaard and Eldrup, 1974). Appropriate source correction was made.

Doppler broadening (DB) measurements were also performed. The DB data were analyzed in terms of the conventional shape parameters $S$ and $W$.

Gas permeation measurements were performed for all membranes with different $\mathrm{PU} / \mathrm{PBDO}$ ratios.

\section{Results and discussion}

The LT spectra were deconvoluted into three components with lifetimes $\tau_{i}$ and intensities $I_{i}$. Subscripts $i=1-3$ are ascribed to $p$-Ps, free $\mathrm{e}^{+}$and $o$-Ps, respectively. The lifetime $\tau_{3}$ and intensity $I_{3}$ are associated with pick-off annihilation of $o$-Ps in free volumes. According to the simple model of Tao and Eldrup (Tao, 1972; Eldrup et al., 1981) for an o-Ps confined in an infinitely deep spherical potential well, there is a correlation between the free-volume hole radius, $R$, and the long-lived lifetime, $\tau_{3}$, in ns

$$
\tau_{3}=0.5\left[1-\frac{R}{R_{0}}+\frac{1}{2 \pi} \sin \left(\frac{2 \pi R}{R_{0}}\right)\right]^{-1},
$$

where $R_{0}=R+\Delta R$, and $\Delta R=1.656 \AA$ (Nakanishi et al., 1987). In terms of this free-volume model, the fractional free volume, FFV, is determined by the product of $I_{3}$ by the cavity volume $V_{\mathrm{f}}=(4 / 3) \pi R^{3}$ and by a material-dependent constant (Liu et al., 1993).

The inclusion of $I_{3}$ on the calculations of FFV from PALS has been recently questioned on the basis of results on temperature dependent studies on a given amorphous polymer (Maurer and Schmidt, 2000; Schmidt and Maurer, 2000). However, above $T_{\mathrm{g}}$, where our measurements were performed, the applicability of the free-volume model seems to be quite acceptable.

In the present study, where polymers with different chemical composition are measured at a constant temperature above $T_{\mathrm{g}}$ and for which appropriate values of the material-dependent normalization parameter are unknown, it is expected that not only $V_{\mathrm{f}}$ but also $I_{3}$ have to be explicitly considered to obtain a reasonable estimate for the variation of total amount of freevolume from PALS results. Accordingly, considering that the PALS method should not be regarded as an absolute but rather a relative measurement technique, we calculate a relative FFV value from the free-volume model, normalized to the PU value, as a characteristic measure.

The values of $R$ and the normalized FFV parameter, calculated for polymers with different amounts of polybutadiene diol (PBDO) are shown in Fig. 1. A continuous decrease is observed for these parameters with increasing PBDO content until about $50 \%$; for higher PBDO content the values remain about constant.

These data are in agreement with previous thermal analysis studies (Zhao and Pinho, 1999) showing two glass transition temperatures $\left(T_{\mathrm{g}}\right)$ for membranes with PBDO content below $50 \%$ due to the coexistence of two phases corresponding to separate domains of the two soft segments (polybutadiene and polypropylene oxide). The two distinct $T_{\mathrm{g}}$ transition temperatures get closer to each other with increasing PBDO content and only one transition temperature is observed for PBDO content above $50 \%$, indicating the formation of a homogeneous phase (full molecular mixing of the two soft segments). Optical microscopy has also shown the presence of these phases.

The dependence of $\mathrm{CO}_{2}$ permeability with $\mathrm{PBDO}$ content is shown in Fig. 2 b. The PU membrane $(0 \%$ PBDO), which is not a bi-soft segment membrane, presents a low $\mathrm{CO}_{2}$ permeability in contrast to the high values of its pore radius and FFV, as determined by LT. The increase up to $100 \%$ in the permeability of the membranes when a low percentage of PBDO is added is not mirrored in the LT parameters that even show a 


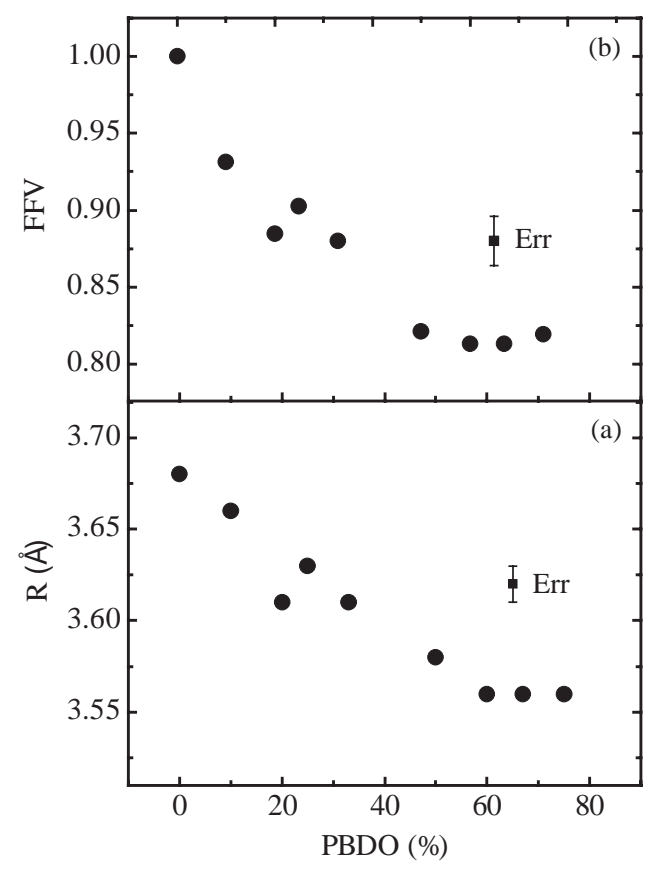

Fig. 1. Variation of the free-volume radius, $R$, (a) and of the relative fractional volume, FFV, normalized to the pure PU polymer (b), with increasing percentage of PBDO.

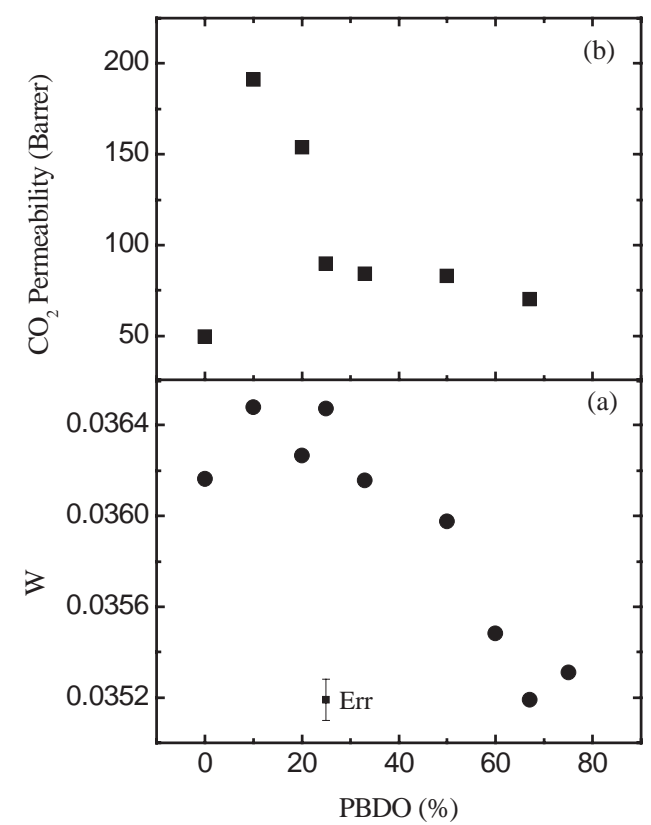

Fig. 2. Variation of the $W$-parameter (a) and the $\mathrm{CO}_{2}$ permeation (b), with increasing percentage of PBDO.

decrease. These results strongly indicate that, besides the free volume probed by $o$-Ps, there are other factors involved in the permeation. Indeed, the interconnectivity and transmissibility between holes may play a dominant role in that process. These features could be drastically changed by reordering with the formation of different free-volume patterns without a significant change in the overall amount of free volume. Comparing now only bisoft segment membranes, the highest permeability is obtained for low PBDO content. The increase in PBDO content favors the tendency for formation of more homogeneous membranes. The lower degree of segregation implies a decrease in the $R$ and FFV values. This picture seems to be supported by DB measurements shown in Fig. 2a, where the $W$-parameter exhibits a maximum just in the concentration range where the gas permeability also has its maximum. This increase may be interpreted by assuming some ordering of polymer chains connected by chemical bounds where positrons can annihilate with high momentum electrons. This ordering could indeed provide an easier path for diffusion between holes. At this PBDO concentration range, the $R$ and FFV values may even decrease.

Results of LT and DB measurements performed on some selected samples as a function of temperature, from room temperature until $50^{\circ} \mathrm{C}$, show no change for samples in the homogeneous phase, whereas an increase of both $R$ and FFV is found for the two-phase range. These data seem to support the picture described above, as in the homogeneous phase no ordering is expected.

In this picture, the fact that the highest values of $R$ and FFV are obtained for the PU membranes, which present low gas permeation, may be considered as not really relevant since, at $0 \%$ PBDO content, we are not dealing with bi-soft segment membranes.

\section{Conclusions}

It is shown that hole size and overall free volume in bisoft segment polymer membranes, as obtained by positron annihilation, are correlated to gas permeation properties. However, the changes observed in those freevolume parameters are of much lower magnitude than the changes found in permeability and it can be concluded that hole size and overall free volume cannot by themselves describe those changes. The free volume seen by the $o$-Ps does not need to be identical to the free volume available for the gas permeation.

The extensive studies on gas permeation (e.g., Nagel et al., 2002) clearly indicate that the high complexity of the phenomena is influenced by many factors not discussed here due to space limitations, e.g., chain flexibility, chemistry at the free-volume walls, diffusion barriers, etc. As the gas permeation requests the actual physical passage of molecules across the polymer membrane, it seems to be also obvious to assume that the interconnectivity, which is not mirrored by the overall amount of the effective free volume seen by 
PALS must play a determining role in the outcome of the permeation features.

It is also not fully surprising that positrons, presenting only a static picture, cannot experience the driving forces due to concentration gradients and the size filtering across pathways through the wobbled interconnections among the holes whereas permeation is a dynamic process. A different diffusion mechanism may take place when the pattern changes from the pure PU to the bisoft system.

The presented work draws attention to the importance of such considerations, and also to the high additional value of Doppler measurements to the lifetime investigations, conventionally applied alone.

Further work along the above lines is in progress.

\section{Acknowledgements}

One of the authors, MFFM expresses thanks to the Instituto Politécnico de Coimbra for partial funds. Additional support through the European Commission HPMF-CT-2000-01038/MCFI-2000-01879 contract is highly acknowledged.

\section{References}

Blackwell, J., Gardner, K.H., 1979. Structure of the hard segments in polyurethanes elastomers. Polymer 20, 13-17.

Cooper, S.L., Tobolsky, A.V., 1966. Properties of linear elastomeric polyurethanes. J. Appl. Sci. 10, 1837-1844.

Dlubek, G., Fretwell, H.M., Alam, M.A., 2000. Positron/ positronium annihilation as probe for the chemical environment of free volume holes in polymers. Macromolecules 33, 187-192.

Dlubek, G., Stejny, J., Lüpke, T.H., Bamford, D., Petters, et al., 2002. Free-volume variation in polyethylenes of different crystallinities: positron lifetime, density, and X-ray studies. J. Polym. Sci. B 40, 65-81.
Eldrup, M., Lightbody, D., Sherwood, J.N., 1981. The temperature dependence of positron lifetimes in solid pivalic acid. Chem. Phys. 63, 51-58.

Flores, K.M., Suh, D., Dauskardt, R.H., Asoka-Kumar, P., Sterne, P.A., Howell, R.H., 2002. Characterization of free volume in a bulk metallic glass using positron annihilation spectroscopy. J. Mater. Res. 17, 1153-1161.

Fridman, I.L., Thomas, E.L., 1980. Morphology of crystalline polyurethane hard segment domains and spherulites. Polymer 21, 388-392.

Jean, Y.C., 1993. Characterizing free volumes and holes in polymers by positron annihilation spectroscopy. In: Advances with positron spectroscopy of solids and surfaces. NATO, Advanced Research Workshop, pp. 563-580.

Kirkegaard, P., Eldrup, M., 1974. Positronfit extended: a new version of a program for analysing positron lifetime spectra. Comput. Phys. Commun. 7, 401-409.

Liu, J., Deng, Q., Jean, Y.C., 1993. Free-volume distributions of polystyrene probed by positron annihilation: comparison with free-volume theories. Macromolecules 26, 7149-7155.

Maurer, F.H.J., Schmidt, M., 2000. Some remarks on the relation between free volume fractions and ortho-positronium lifetimes in amorphous polymers. Radiat. Phys. Chem. $58,509-512$.

Nagel, C., Günther-Schade, K., Fritsch, D., Strunskus, T., Faupel, F., 2002. Free volume and transport properties in highly selective polymer membranes. Macromolecules 35, 2071-2077.

Nakanishi, H., Wang, S.J., Jean, Y.C., 1987. Microscopic surface tension studied by positron annihilation. In: Sharma, S.C. (Ed.), Positron Annihilation Studies of Fluids. World Science, Singapore, pp. 292-298.

Schmidt, M., Maurer, F.H.J., 2000. Relation between free volume quantities from PVT-EOS analysis and PALS. Polymer 41, 8419-8424.

Semaan, M.E., Quarles, C.A., Nikiel, L., 2001. Positron annihilation spectroscopy of carbon black/silica filled rubber polymers. Mater. Sci. Forum 363-365, 278-280.

Tao, S.J., 1972. Positronium annihilation in molecular substances. J. Chem. Phys. 56 (11), 5499-5510.

Zhao, C-t., Pinho, M.N., 1999. Design of polypropylene oxide/ polybutadiene bi-soft segment urethane/urea polymer for pervaporation membranes. Polymer 40, 6089-6097. 